\title{
Diagnosis of Infectious Bronchitis: An Overview of Concepts and Tools
}

Workshop: Infectious Bronchitis (IB) in the Brazilian Poultry Industry

\section{Author(s)}

Villarreal LYB

Intervet Schering Plough Animal Health Coronavirus Research Group

\section{Mail Address}

Laura Yaneth Villarreal Buitrago

Av. Sir Henry Wellcome 335

Moinho Velho

06.714-050. Cotia, SP, Brazil.

E-mail: laura.villarreal@sp.intervet.com

Keywords

Antibodies, diagnosis, IBV, typing.

\section{ABSTRACT}

Infectious bronchitis (IB) casues multi-systemic infection in chickens with signs similar caused by other poultry pathogens and thus a high diagnostic accuracy can only be achieved by s series of laboratory assays. This article reviews in a brief way the traditional virus assays such as embryo innoculation, tracheal rings and virus neutralization assays for the direct detection of Avian infectious bronchitis virus (IBV) and methods based on gene molecular biology and some assays for the detection of anti-IBV antibodies, including ELISA.

A critical view on each technique is also provived by the author.

\section{INTRODUCTION}

Recognized since 1931, the chicken infectious bronchitis (IB) can be defined as an infectious-contagious disease that mainly affects the renal, reproductive and enteric systems of breeders, layers and broilers, caused by a great diversity of avian infectious bronchitis virus (IBV) types of worldwide distribution.

The importance of IB lies in the economic losses caused by reduced egg production, worse egg internal and external quality, presence of silent layers, infertility, growth delay, increase in the susceptibility to secondary infections, and, in some cases, moderate to severe mortality.

\section{LABORATORY DIAGNOSIS}

Although IBV is a virus that causes considerable economic losses in poultry, the clinical signs of IB are not specific. Therefore, it is necessary to use tools to identify IBV when there are clinical problems in the field. In general, IB can be diagnosed by detecting the virus itself (or parts of it) or by the specific antibody responses. The choice of the best test diagnosis test and its subsequent interpretation may be very difficult and confusing.

Factors that influence the successful detection of IBV, according to De Wit (2000):

a) Time elapsed between the beginning of the infection and sampling: the upper respiratory tract is the primary site of IBV replication, which is followed viremia, causing the virus to disseminate to other tissues. All IBV types can be isolated from the upper respiratory tract, with the highest concentration found in the trachea during the first 3 to 5 days post-infection (p.i). After this period, virus titer falls quickly in the second week, below the detection levels.

A complicating factor from the diagnosis point of view is the definition of which organs and how many birds are virus carriers (be it from vaccine or field origin). There are possible explanations for long-term isolations 
or reverse-excretion of the inoculated virus, such as continuous cross-infection among infected or vaccinated flocks. The two main sites of the virus persistence are the cecal tonsils and kidneys.

b) Chicken immunity level at the time of infection: The level of acquired immunity at the time of infection has the highest influence on the period and quantity of IBV that can be detected. The presence of maternal antibodies does not reduce the level of re-isolation of challenge virus in the trachea and in the kidney after the challenge of 2-day-old chicks.

c) Number of sampled chickens: taking fewer samples than required decreases the chances of detecting IBV infection.

d) Selection of organs (samples): When acute breathing symptoms are predominant, the respiratory tract is the preferred site for sample collection. Kidneys, cecal tonsils, and cloaca are sampled preferably when there are chronic infections or infections in vaccinated chickens, such as layers and breeders, in which small amounts of virus are expected in the respiratory tract.

e) Sample quality: The samples should be quickly refrigerated to preserve virus viability. If the freezing or refrigerating is not possible, samples should be place into $50 \%$ glycerin, where IBV remains viable for many days.

f) Bird genetics: Several studies indicate that genetic aspects may influence the susceptibility to IBV. Different lines of chickens present variable mortality after the inoculation of IBV alone or in co-infection with E. coli.

g) Virus isolation (multiplication and detection of infectious (BV): virus isolation can be laborious, time-consuming, and expensive. Additionally, the classic method of isolation may require several passages in embryonated eggs until embryonated mortality occurs or other signs are detected in the embryos.

IB is one of the most important causes of economic losses in poultry farming, and may be related to breathing problems, nephritis, and reduced egg production and quality. However, these symptoms are not specific of IB, and therefore, diagnostic tools are necessary to identify infections by IB when clinical problems are observed in field. These diagnostic tools may include the classification of types identified during isolation, with the purpose of selecting the vaccination program that will provide the best protection of the flocks.

A review on the diagnostic techniques applicable to IB can be found in Di Fábio \& Villarreal (2009).

\section{INDIRECT DIAGNOSIS}

\section{Detection of antibodies}

Infections by IBV can be diagnosed by the detection or by increase of IBV specific-antibody titers. In general, paired serum sampling is necessary to correlate the clinical problem with IBV infection. The first sample is collected at the beginning of the disease and the second sample, 4 weeks later.

Some factors may influence the success of IBV antibody detection, such as age at the time of infection/ vaccination (the degree of immune humoral response after infection or vaccination may decrease when the infection happens at a very early age, because many chicks are not yet immune-competent), the presence of maternal antibodies at the time of infection/ vaccination (this may delay or reduce the serologic response to vaccination or to infection); presence of immunity at the time of infection/vaccination (the sensitivity of tests to detect antibodies may be much lower in vaccinated chickens as compared to nonvaccinated chickens); number of sampled chickens (the number of chickens that should be sampled to detect seroconversion depends on the prevalence of the disease and the test sensitivity); cross reactions between serotypes, and occurrence of new and unexpected types of IBV (De Wit, 2000).

\section{ELISA}

It is an immunoenzymatic method and its automation enables the detection and titration of antibodies in a large number of serum samples. Most of the ELISA tests are generic for IBV; in other words, they do not differentiate serotypes. This is due to the fact that the surface of the plate in which antigen-antibody reaction takes place is impregnated with the viral suspension in its complete form. The reaction is positive when any IBV strain is present. It detects IgG, and therefore, it is an indicator of humoral immunity, enabling the analysis of post-vaccination and infection responses (in adult birds).

\section{Virus neutralization assay}

It detects antibodies produced by the $\mathrm{S} 1$ protein fraction; and therefore, it is a serotype-specific test. It can be carried out in cell cultures or in tracheal ring 
cultures. The reading has the same principle as virus isolation in embryonated eggs, cell cultures and tracheal ring cultures, in other words, it detects embryo alterations, cytopathic effect, and ciliostasis. This assay requires sera with no cross reaction with other serotypes. Neutralizing antibodies are highly specific and responsible for the serum-protecting effect. They target important antigens of the viral surface that play a role in the process of cell absorption. A disadvantage of this test is the lack of standardization among the different virus neutralization systems, making it difficult to compare the results among different laboratories.

\section{Immunodiffusion in agar gel}

This test requires neither many pieces of equipment, nor specific facilities. This test requires the inclusion of a positive control serum to differentiate non-specific precipitation bands from specific IBV precipitation bands. Although this test is quite simple, it is not standaridized for IB (De Wit, 2000).

\section{Hemagglutination inhibition test (HI)}

IBV is not naturally hemagglutinating, and requires previous treatment with type $C$ phospholipase enzyme to expose hemagglutinin, which makes this test difficult to perform and to standardize. Hemagglutinationinhibiting antibodies are induced primarily against S1 spike protein. $\mathrm{HI}$ test usually detects first antibodies between 1 and 2 weeks after infection. The $\mathrm{HI}$ test is serotype-specific when used to detect antibodies after a single inoculation.

$\mathrm{HI}$ serotype specificity is much lower after IBV reinfection, especially when the second or subsequent serotype is heterologous (De Wit, 2000).

\section{DIRECT DIAGNOSIS}

\section{Isolation in embryonated eggs}

The characteristic effects caused by IBV in chicken embryonated eggs are the most classic signs for the diagnosis of this virus, and have successfully been used since the beginning of the studies on IB.

Such effects include embryo dwarfism, curling, hemorrhages, and death. The lesions vary according to sample/type, and their intensity increase as the number of egg passages increase.

In this test, 9- to 10-day-old chicken embryonated eggs are inoculated in the allantoidal cavity with 0.1 to $0.2 \mathrm{~mL}$ of a field sample (macerate of organs, enteric content, tracheal and cloacal swabs, all prepared as suspensions, added antibiotics, and filtered in $0.22 \mathrm{~m}$ - porosity membranes) and incubated again for a period of up to four days, after which the allantoidal liquid is collected, and submitted to new passages, and then the embryos are morphologically analyzed. Maximum IBV titer is reached 1 to 2 days post-inoculation (except for those samples which do not adapt to embryonated eggs)

The chicken embryonated egg is are an effective model for isolation of IBV field samples as it can be used with most field strains; however, the disadvantage is that usually three successive passages are needed for the manifestation of characteristic lesions in embryos, which delays diagnosis. Additionally, isolation in eggs may be reduced, for instance, when IBV is inactivated due to bad preservation. Another possible cause for virus isolation failure is the co-infection with other virus that prevents IBV replication in the embryos.

In addition, if higher specificity is required, a confirmation test needs to be conducted, such a virus neutralization or reverse transcription followed by polymerase chain reaction (RT-PCR).

\section{Cultivation in trachea rings}

This technique uses trachea rings of SPF embryos at 19 to 20 days of incubation. The rings obtained from embryo tracheas are individually placed into test tubes containing cell culture media and antibiotics, and incubated at $37{ }^{\circ} \mathrm{C}$ in a rotating cultivation system for 48 hours, and only rings with more than $50 \%$ ciliary motility are used.

After this period, the culture media is removed and $0.1 \mathrm{~mL}$ of suspension of the sample to be tested is added, followed by 1 hour of incubation for viral adsorption. One $\mathrm{mL}$ of culture media, is then added, and the sample is incubated again.

At 24, 48, 72 and 96 hours after inoculation, the trachea rings are observed in inverted optical microscope, evaluating the ciliary motility, which should decrease due to replication of IBV (Epiphanio et al., 2002).

Although this is an efficient preventive technique, it has the disadvantage of not being sensitive for IBV field samples that do not present tropism for the respiratory tract, and therefore, it may yield falsenegative results.

It should be taken into account that ciliostasis can also be induced by many other agents, requiring the confirmation of IBV by other specific methods.

\section{Methods based on nucleic acids}

IBV detection based on the evidencing of specific viral RNA presence by the technique of reverse 
transcriptase reaction followed by the polymerase chain reaction (RT-PCR) has increased in the last years, because of its accuracy for diagnosis and classification of types. Its low implementation and execution costs have allowed a large number of public and private laboratories to use routinely.

Shortly, the RT-PCR technique firstly produces DNA from IBV RNA using small DNA "probes" called primers. The produced DNA, called complementary DNA, is amplified billions of times by the polymerase chain reaction, generating a double-stranded DNA fragment, measured in pairs of bases limited by primers and, afterwards, the specific DNA fragment is detected by electrophoresis.

In order to perform screening diagnosis using RTPCR method, the genomic regions that are highly preserved within a certain kind of pathogen needs to be selected to allow primers to start the amplification of specific sequences present in the largest possible diversity of types of the agent to be detected. In the case of IBV, the regions of the genome commonly use for screening diagnosis are the gene that encodes the $\mathrm{N}$ nucleoprotein and a region located at the end of the IBV genome that does not encode any proteins, but has a function in the replication of the viral genome and that is called $3^{\prime}$ untranslated region (3' UTR). In other words, RT-PCRs that use these regions as a target are able to diagnose virtually any IBV serotype or genotype. However, this does not allow differentiating IBV samples / strains, which requires the selection of a region that allows detecting differences among such samples. In this case, the gene of the $S$ glycoprotein spike, which is specific of each serotype and genotype, can be use, as this protein is the one that suffers the highest selection pressure from the immune system.

Therefore, it is possible in RT-PCR to use primers that target the $S$ gene to specifically detect this or that type of IBV, or that produce DNA, allowing differentiation by DNA sequencing. Although RT$P C R$ is a highly sensitive and specific technique, conventional and reference viral diagnosis tests should not be left aside. The association of the results obtained using different techniques allow a more accurate and objective diagnosis. In addition, RT-PCR does not differentiate infectious from non-infectious viral particles.

Finally, the laboratory diagnosis methods for IBV detection are mandatory both to measure the incidence of this virus, as well as to determine which are the IBV strains circulating among Brazilian poultry in order to prevent and to control infectious bronchitis.

\section{REFERENCES}

De Wit JJ. Detection of infectious bronchitis virus. Technical review. Avian Pathology 2000; 29:71-93.

Di Fábio J, Villarreal LYB. Bronquite infecciosa das galinhas. In: Berchieri Jr A, Silva EN, Di Fabio J, Sesti L, Zuanaze MAF. Doenças das aves. Campinas: FACTA; 2009. p. 631-648.

Epiphanio EOB, Martins NRS, Resende JS, Pinto RG, Jorge MA, Souza MB, Caccioppoli J, Cardozo RM. Resultados preliminares da utilização de cultivos de anéis de traquéia para o estudo de estirpes brasileiras do vírus da bronquite infecciosa das galinhas. Arquivos Brasileiros de Medicina Veterinária e Zootecnia 2002; 54:2. 